九州大学学術情報リポジトリ

Kyushu University Institutional Repository

\title{
A Study on Electrical Wire and Floating Tower Interaction for an Offshore Overhead Power Transmission System
}

Luo, Ruo

Interdisciplinary Graduate School of Engineering Sciences, Kyushu University

Zhu, Hongzhong

Research Institute for Applied Mechanics, Kyushu University

$\mathrm{Hu}$, Changhong

Research Institute for Applied Mechanics, Kyushu University

https://doi.org/10.5109/4738552

出版情報: Proceedings of International Exchange and Innovation Conference on Engineering \& Sciences (IEICES). 7, pp. 13-18, 2021-10-21. 九州大学大学院総合理工学府

バージョン:

権利関係: 


\title{
A Study on Electrical Wire and Floating Tower Interaction for an Offshore Overhead Power Transmission System
}

\author{
Ruo Luo ${ }^{1 *}$, Hongzhong $\mathrm{Zhu}^{2}$, Changhong $\mathrm{Hu}^{2}$ \\ ${ }^{1}$ Interdisciplinary Graduate School of Engineering Sciences, Kyushu University, Fukuoka, Kasuga, 816-8580, Japan \\ ${ }^{2}$ Research Institute for Applied Mechanics, Fukuoka, Kasuga, 816-8580, Japan \\ *Corresponding author email: luoruo2019@riam.kyushu-u.ac.jp
}

\begin{abstract}
Numerical simulation has been carried out to investigate the interaction between electrical wire and floating tower for a new proposed offshore overhead power transmission system. The motion of the TLP-type floating tower is calculated by a potential flow theory method and dynamics of the wire is calculated by a lumped mass method. The numerical model is validated by comparing to available experimental data. In this study, a triangle TLP is newly designed for the floating tower and the interaction of the wire and the floating tower is investigated by a series of numerical simulation. It is found that the wire-floating tower interaction is significantly affected by the wave and wind direction, which should be carefully considered in the safety assessment of the system.
\end{abstract}

\section{Keywords: Overhead power transmission; Lumped parameter method; Wind-wave misalignment}

\section{INTRODUCTION}

Offshore wind power generation shows a potential growth in wind power market [1]. Even though systemic research and engineering development only started at the beginning of this century [2-5], many countries have scheduled offshore power plants as part of their national decarbonization projects. China has made a national target [6] which calls for $5 \mathrm{GW}$ of offshore capacity by 2020. European union (EU) countries have been improving the capacity of offshore power generation and a well-developed supply chain has been established around the North Sea [7]. New programs have been carried out by the World Bank Group to speed up the deployment of offshore wind power in emerging markets, which shows a strong confidence in foresight of this industry.

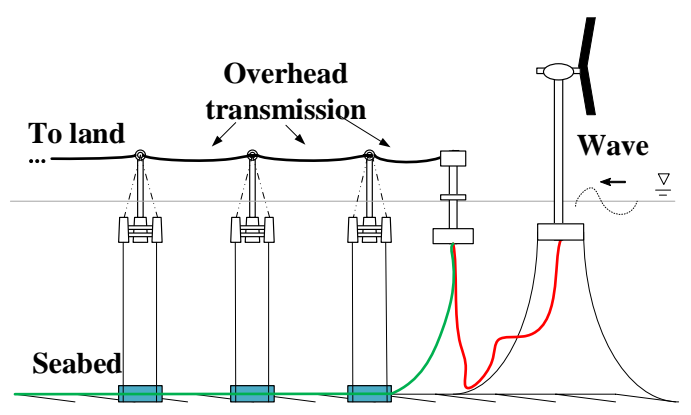

Fig. 1. Overview of offshore power transmission types

Currently, submarine cables are the practical method for long distance offshore power transmission. Though the cost of cables had been quite reduced with the development of material technology, the maintenance cost and electrical loss in transmission can reach a high level in offshore work condition because of the complex seabed environment [8-9]. To provide an alternative power transmission method, overhead power transmission system is proposed by our research group. The overview of the proposed system is shown in Fig.1, submerged tension leg platform (TLP) towers are used to locate the transmission path and hold the overhead conductor wire. Compared with submarine cable method, the overhead transmission method has the potential to reduce the power transmission cost because of its relatively low power loss and simple installation/ maintenance procedure.

In this study, numerical simulation is carried out based on potential flow theory, and its reliability is verified by using the data from previous experimental study [10]. Then, a novel TLP is designed by taking account of extreme environmental condition. Assessment of the system is made by investigating the parameters of the TLP and wire.

\section{NUMERICAL MODEL}

To make simulation under variable environmental conditions, numerical model of TLP-wire system is established and numerical results are compared to the model experiment results in this section.

\subsection{TLP hydrodynamic model}

Regarding the floating TLP tower as an oscillating system, the governing equation can be expressed as:

$$
[M+A(\infty)] \ddot{\xi}+\int_{0}^{t} K(t-\tau) \dot{\xi}(\tau) \mathrm{d} \tau=F
$$

Where $M$ is the mass of TLP, $A(\infty)$ is the added mass in water at infinite frequency, $K(t)$ is the retardation function of hydro-damping. Term $F$ is the forces including loads from wind-wave excitation, restoring forces and damping forces. The hydrodynamic forces including exciting and radiation force can be obtained by the hydrodynamic software such as HydroSTAR[11] and WAMIT[12] or other open-sources[13], by integrating the pressure of water over the wetted surface of structure obeying potential-flow theory.

The viscous damping of TLP structure mainly contains potential damping and "vortex shedding" damping. As the potential damping can be expressed by retardation function $K$ mentioned above, vortex shedding damping is expressed as[14]:

$$
f(\dot{\xi})=-0.5 \rho C_{D} A|u| u
$$

Term $A$ and $C_{D}$ are the projected cross-sectional area and drag coefficient determined by Reynolds number, respectively. $\rho$ is the water density. These damping force and torque can also be calculated by surface integral. Considering linear elastic mooring line with a strength limit, the hybrid linear elastic spring model[15]can be 
expressed as:

$$
F_{m}=\left\{\begin{array}{cc}
0 & \left(l_{m} \leq d_{m}\right) \\
k\left(l_{m}-d_{m}\right) & \left(l_{m} \geq d_{m}\right)
\end{array}\right.
$$

Term $l_{m}$ and $d_{m}$ are the exact length and nominal length of tendon, respectively. By frame transformation to TLP tower, dynamic damping term $c$ from mooring lines can be calculated as:

$$
c_{i}=2 \zeta \sqrt{k_{i}^{\prime} m_{i}}
$$

Where $k_{i}^{\prime}$ is the eigen stiffness in each DOF and $\zeta$ is the structural damping ratio of the mooring lines.

\subsection{Lumped parameter model for conductor wire}

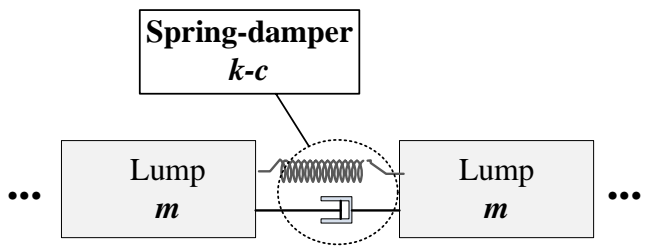

Fig 2. Wire model by lumped parameter method

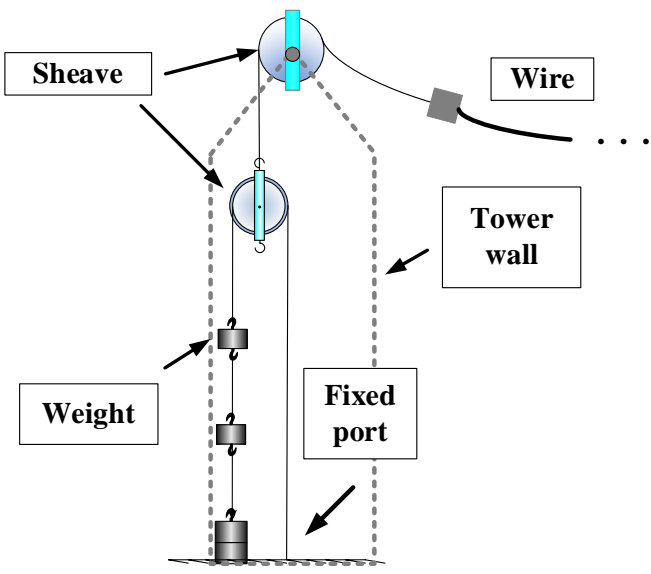

Fig 3. Sheave system to connect wire and tower

The conductor wire can be regarded as a flexible line and modeled by lumped parameter system[16], it can be divided by several mass units connected by linear elastic units. Wire is divided into lumps of equal mass which are connected by spring-damper system to express its flexibility. A partial view of lumped-mass system can be shown in Fig 2.

To suppress wire's motion and internal tension, sheaves are used to adjust wire's axial motion[20]. The weights to adjust wire's tension are used to restricted vertical motion and will change according to wire's axial motion. In Simulink, the block "pulley" can be used to express this control system. The overview of wire-sheave system can be shown as Fig.3.

\subsection{Modeling Wind and wave loads}

\subsubsection{First order wave load}

In real sea environment, the sea state contains varies of wave frequencies, and it can be characterized by wave spectrums such as JONSWAP, ITTC or PiersonMoskowitz. In this paper, the Bretschneider spectrum[17] which can be expressed as following is applied to the simulation:

$$
s(\omega)=C \omega^{-5} \exp \left(-\mathrm{D} \omega^{-4}\right)
$$

Where the sea state parameters $C$ and $D$ are given by:

$$
C=\frac{5 \pi^{3} \omega_{P}^{4} H_{S}^{2}}{16} \quad D=\frac{5 \omega_{P}^{4}}{4}
$$

Here $H_{S}$ and $\omega_{P}$ represent the significant wave height and modal wave frequency which can be summarized from observation records, respectively. The 1st wave excitation force respect to the wave frequencies can be calculated by:

$$
\begin{gathered}
F_{e x}(t)=|\Gamma(\omega)| \cdot a(\omega) \cdot \cos \left(\omega t+\angle \Gamma(\omega)+\varepsilon_{\omega}\right) \\
a(\omega)=\sqrt{2 s(\omega) \Delta \omega}
\end{gathered}
$$

$\Gamma(\omega)$ and $\angle \Gamma(\omega)$ are the amplitude and phase of the force RAOs according to frequency component, respectively. $\Delta \omega$ is the constant difference between the successive frequencies in wave spectrum $s(\omega)$. To simulate the irregular wave environments, a random phase $\varepsilon_{\omega}$ is generated by random function.

\subsubsection{Second order wave load}

The wave drift load and sum frequency load are second order wave loads that act on objects subject to irregular waves, and the values can be expressed by:

$$
\begin{gathered}
F_{d}=\sum_{i=1}^{N} \operatorname{Re}\left[Q _ { d } ( \omega _ { i } , \omega _ { j } ) a ( \omega _ { i } ) a ( \omega _ { j } ) \operatorname { e x p } \left(\left(\omega_{i}-\omega_{j}\right) t+\right.\right. \\
\left.\angle \Gamma\left(\omega_{i}\right)-\angle \Gamma\left(\omega_{j}\right)\right) \\
F_{d}=\sum_{i=1}^{N} \operatorname{Re}\left[Q _ { d } ( \omega _ { i } , \omega _ { j } ) a ( \omega _ { i } ) a ( \omega _ { j } ) \operatorname { e x p } \left(\left(\omega_{i}-\omega_{j}\right) t+\right.\right. \\
\left.\left.\angle \Gamma\left(\omega_{i}\right)-\angle \Gamma\left(\omega_{j}\right)\right)\right]
\end{gathered}
$$

$Q_{d}$ and $Q_{d}$ are figured out by QTF calculation through software HydroSTAR. Using the same difference method in the above section, the $2^{\text {nd }}$ order wave load can be imported in the simulation as a real-time input.

\subsubsection{Wind load}

In the interaction of wire and wind, the drag force from resistance and lift force from vortex inducing should be accounted in wire safety assessment. Considering the coupled motion of wire and tower can affect the aero forces around, wake oscillation model[18,19] is applied to express the wind loads. Load coefficients and wake oscillation factor $q$ which are used to describe the vortex around the wire are given by:

$$
\begin{gathered}
C_{x}=-C_{D L} \frac{v}{u^{2}} \dot{y}+C_{M} \frac{v}{u^{2}} v_{x}-\alpha C_{D L}^{2}\left(1-\frac{\dot{x}}{u}\right)\left|1-\frac{\dot{x}}{u}\right| \\
C_{y}=C_{D L} \frac{v}{u^{2}}(u-\dot{x})-C_{M} \frac{v}{u^{2}} \dot{y} \\
\frac{d^{2} q}{d t^{2}}+\epsilon \omega_{s}\left(q^{2}-1\right) \frac{d q}{d t}+\left(\omega_{s}^{2}-\frac{\kappa}{D} \dot{x}\right) q=\frac{A}{D} \dot{y}
\end{gathered}
$$

Where $u$ is the free wind speed assumed flowing in $\mathrm{x}$ direction, $v=\sqrt{(u-\dot{x})^{2}+\dot{y}}$ is the relative velocity, $D, A, \kappa$ and $\epsilon$ are the tuning parameters determined by wire cross-sectional shape. Considering wires of circular cross-section, drag coefficient $C_{M}$ and dynamic lift coefficient $C_{D L}$ can be defined as:

$$
C_{D L}=0.5 q C_{L 0} \quad C_{M}=1.1
$$

In irregular wind-wave condition, the motion of wire is 
determined by the effect from both wind and TLP tower. And oscillation factor $\mathrm{q}$ affects the bending moment of wire since the lift wind load is mainly relative with it.

\subsection{Validation with experiment}

To verify the reliability of the numerical model for interaction of TLP and tower, previous experiments[10] condition of TLP-wire model in regular wave condition was applied to present numerical model, and comparison of simulation and experiment is shown in Fig 4. It is worthy to notice that the value in $Y$ label is the ratio of wire sag $D$ to regular wave height $H$. Good agreements can be obtained on wire motion as seen in Fig.4. The present numerical model is validated in simulation of the wire-tower interaction.

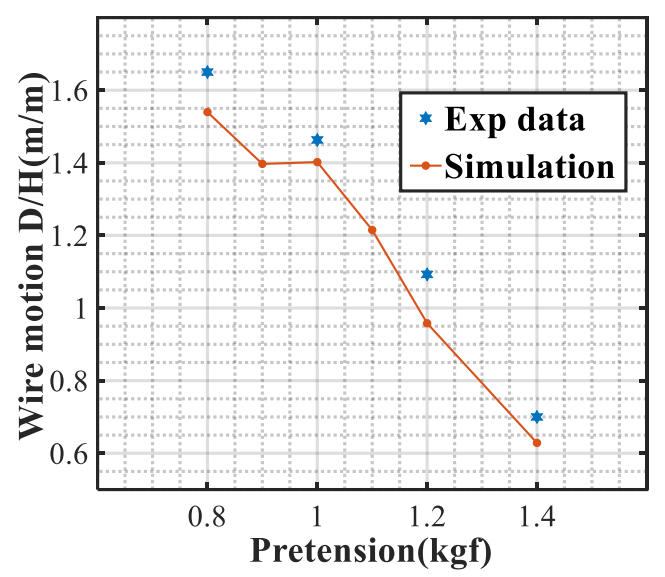

Fig 4. Comparison of wire motion

\section{NUMERICAL SIMULATION OF A NEWLY DESIGNED TLP IN EXTREME SEA CONDITION}

\subsection{TLP tower design}

In this study, a novel TLP tower was designed to be more adaptable to extreme environment condition. Since hydrodynamic performance of TLP is mainly determined by its mooring stiffness and buoyancy, a submerged 3-leg TLP (see in Fig.8) was taken into consideration for overcoming installation difficulties in complex seabed condition and reducing wave loads. The main part of volume is concentrated at leg and center columns to suppress yaw motion by dispersing wave loads.

Since the system of TLP and the overhead conductor wire should avoid resonance, its design should be considered according practical working condition. Considering the added-mass distribution and restoring force of TLP tower, the natural frequencies of the platform in heave, roll and pitch should be designed in high-frequency region, while the natural frequencies of the floating tower in surge, sway and yaw should be designed in a low-frequency level. The frequencies of TLP can be designed as:

$$
\begin{aligned}
& \left(\omega_{x}, \omega_{y}, \omega_{\psi}\right) \leq \omega_{L} \\
& \left(\omega_{z}, \omega_{\varphi}, \omega_{\theta}\right) \geq \omega_{H}
\end{aligned}
$$

Here $x, y, \psi$ represent the motion in surge, sway and yaw, term $z, \varphi, \theta$ represent the motion in heave, roll and pitch, respectively. $\left[\omega_{L}, \omega_{H}\right]$ is the expected range of environmental wave frequencies.

Since the effect from wire to TLP tower is relatively small, pretension of wire $\left[T_{0}, T_{\text {Max }}\right]$ should be satisfied as below according to the TLP properties[21]:

$$
\begin{gathered}
f_{0}=\frac{\pi \sqrt{T_{0} / m_{e}}}{S_{e}} \geq \omega_{H} \\
f_{D H}=\frac{\pi \sqrt{T_{E} / \alpha_{E} m_{e}}}{S_{e}}<\min \left(\omega_{z}, \omega_{\varphi}, \omega_{\theta}\right) \\
f_{D L}=\frac{\pi \sqrt{\frac{T_{m a x}}{g}\left(g-2 \omega_{M}^{2} R_{M} H_{S}\right) / m_{E}}}{S_{e}} \geq 2 \omega_{M} \\
\frac{T_{0}}{g}\left(g+\frac{m_{W}+m_{e}}{m_{e}} \omega_{M}^{2} R_{M} H_{S}\right) \leq \frac{T_{E}}{\alpha_{E}}
\end{gathered}
$$

Where $T_{E}$ and $\alpha_{E}$ represent the tensile strength and preset safety ratio of the wire, term $m_{E}$ and $\omega_{M}$ are the wire's equivalent unit load in high wind speed and modal wave frequency in extreme environment, respectively. Obviously, the wire tension range $\left[T_{0}, T_{\text {Max }}\right]$ are highly relative to TLP motion according to (21) and (22). $R_{M}$ is the expected response amplitude operator(RAO) of TLP and can be given by:

$$
R_{M}=\frac{\left|\Gamma\left(\omega_{M}\right)\right|}{\sqrt{\left(-\omega_{M}^{2} m+k\right)^{2}+\left[\omega_{M} B\left(\omega_{M}\right)\right]^{2}}}
$$

$B$ is the added hydro damping respected to retardation function $K(\omega)$.Noticeably, the expected TLP motion is given out by assuming adjacent TLPs are restricted to in-phase motion.

Based on above assumption, relationship of the design frequencies is shown in Fig.5 in which wire's design frequency range is set to leave TLP's natural frequencies and the low bondage $f_{D L}$ is set as double of the modal frequency to avoid united resonance of the TLP tower and the wire. 


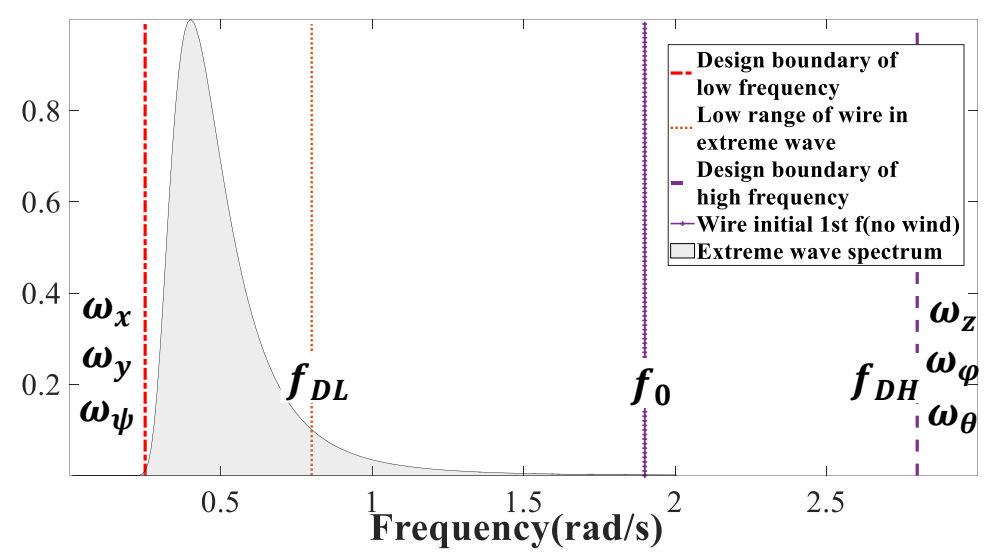

Fig 5. Design frequency for TLP and wire

Table 1. Properties for the newly designed TLP tower

\begin{tabular}{|c|c|}
\hline Property & Value \\
\hline $\begin{array}{l}\text { Total mass } \\
\text { (no water } \\
\text { injected) }\end{array}$ & $2.12 \times 10^{5} \mathrm{~kg}$ \\
\hline Displacement & $620 \mathrm{~m}^{3}$ \\
\hline $\mathrm{COG}$ & {$[0,0,-16.5](\mathrm{m})$} \\
\hline Inertia radius & {$[7.06,7.06,9.36](\mathrm{m})$} \\
\hline Side-length & $20.8 \mathrm{~m}$ \\
\hline Tower height & $40 \mathrm{~m}$ \\
\hline$\left(\omega_{x}, \omega_{y}, \omega_{z}\right.$ & {$[0.18,0.18,2.81,3.25,3.25,0.24]$} \\
\hline$\left.\omega_{\varphi}, \omega_{\theta}, \omega_{\psi}\right)$ & $(\mathrm{rad} / \mathrm{s})$ \\
\hline Wire type & $\operatorname{ACSR} 410 \mathrm{~mm}^{2} \times 3$ \\
\hline Span & $300 \mathrm{~m}$ \\
\hline Water depth & $120 \mathrm{~m}$ \\
\hline $\begin{array}{l}\text { Significant } \\
\text { wave height }\end{array}$ & $14 \mathrm{~m}$ \\
\hline $\begin{array}{l}\text { Modal wave } \\
\text { period }\end{array}$ & $16 s$ \\
\hline $\mathrm{U}_{\text {Wind }}$ & $50 \mathrm{~m} / \mathrm{s}$ \\
\hline $\begin{array}{l}\text { Wave } \\
\text { direction }(\beta)\end{array}$ & $\left(0^{\circ}, 30^{\circ}, 60^{\circ}, 90^{\circ}\right)$ \\
\hline $\begin{array}{l}\text { Wind } \\
\text { direction }\end{array}$ & $90^{\circ}$ \\
\hline
\end{tabular}

Based on above assumption, relationship of the design frequencies is shown in Fig.5 in which wire's design frequency range is set to leave TLP's natural frequencies and the low bondage $f_{D L}$ is set as double of the modal frequency to avoid united resonance of the TLP tower and the wire.

The above frequency properties are determined by extreme environmental condition. A sea state of level 9 (percentage probability $\leq 1 \%$ ) $[16,18]$ with a wind speed $50 \mathrm{~m} / \mathrm{s}$ and a significant wave height $14 \mathrm{~m}$ was applied into the simulation as extreme condition. Considering this situation for the water depth of $120 \mathrm{~m}$, the span distance between TLPs was set as $300 \mathrm{~m}$. According to the equation(19)-(22), the wire of ACSR 410 $\mathrm{mm} 2$ (tensile strength $T_{E}=136 \mathrm{KN}$ ) was employed as the power conductor in order to serve for a moderate wind farm with a capacity of $90 \mathrm{MW}$ (three-phase power transmission under $66 \mathrm{kV}$ ). The displacement of TLP was set as $620 \mathrm{~m}^{3}$ considering a safety factor of 3 in tendons under wind-wave loads. The overall properties are shown in Table 1.

\subsection{Simulation in extreme environmental condition}

To check the effect of wind-wave misalignment on conductor wire, conditions of different wave directions and a $90^{\circ}$ incident wind was employed into simulation as Fig 6.

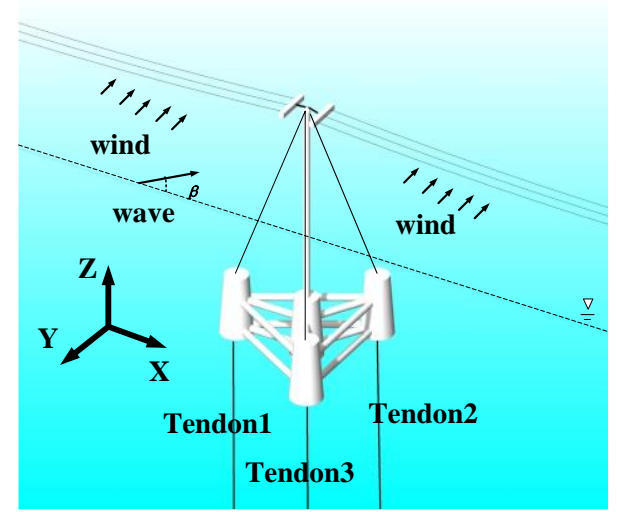

Fig 6. Schematic of TLP tower in wind-wave condition (incident angle)

Fig. 7 shows the distribution of the TLP motion, in which the maximum values of motion appear at the wave direction of $30^{\circ}$ or $60^{\circ}$. It is noticeable that the maximum offset of yaw motion appears at $\beta=30^{\circ}$ 


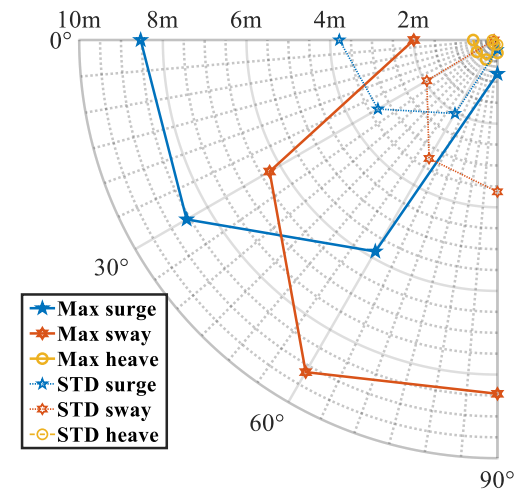

(a) Translation

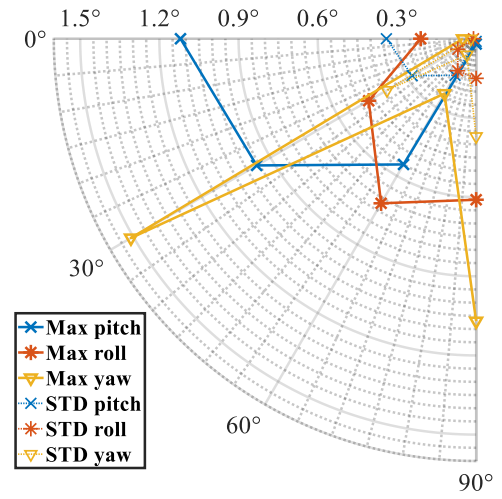

(b) Rotation

Fig 7. Tower motion distribution

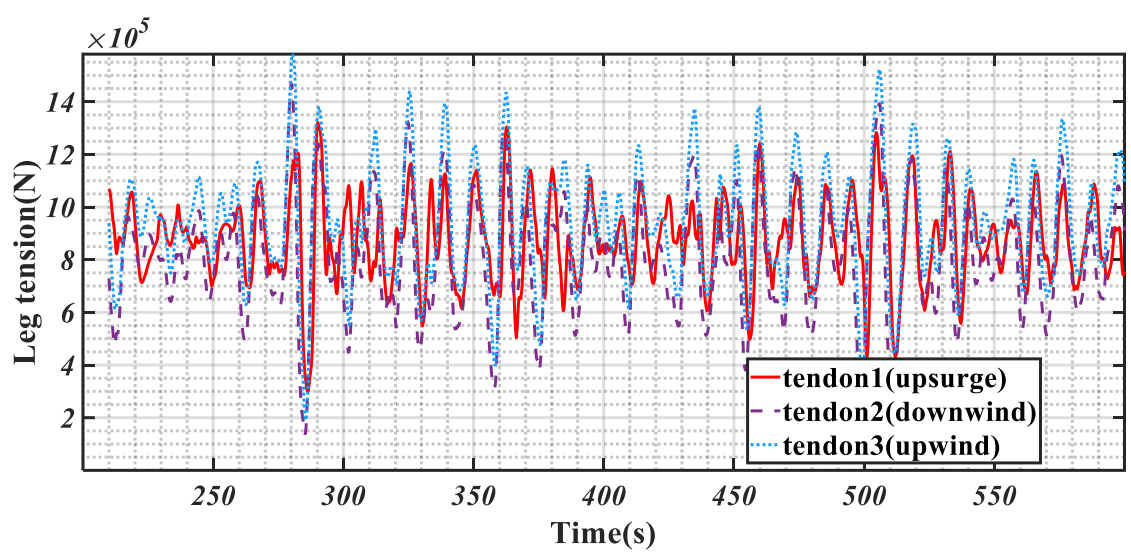

Fig 8 . Time series of tension for tendon legs $\left(\beta=30^{\circ}\right)$

which shows a significant rise than the other wave directions.

The simulation results of 3 tendon legs' tension are shown in Fig.8 and Fig.9.

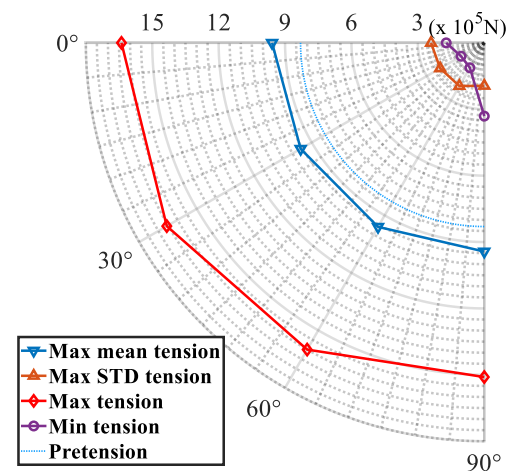

Fig 9. Leg tension in different wind-wave direction

From Fig.8 and Fig.9, it can be seen that the maximum tension, minimum tension, and maximum standard deviation(STD) of tendon tension appear at the downwind tendon (tendon 3 ) at the wave direction of $\beta$ $=30^{\circ}$, where a large yaw motion is found which may lead to tendon's instability in wave and current (Mathieu instability). The maximum tendon tension, which is about double of the pretension, shows small variation with the wave direction which indicates the little effect from wire. In the design process, the tension distribution for different wave directions should be carefully studied in design of mooring system.

To study the wire safety in wind-wave environment, wire's tension is traced for study. Wire's tension is shown in Fig.10, both the maximum tension and STD tension appear at the wave direction of $0^{\circ}$ and the maximum tension reach a value of $1.05 \times 10^{5} \mathrm{~N}$ which is about $3 / 4$ of the tensile strength. This may be a dangerous situation, however this situation only occurs when wind-wave misalignment angle is $90^{\circ}$ which rarely happens in opensea environment. Therefore, present TLP system is acceptable in wire safety evaluations.

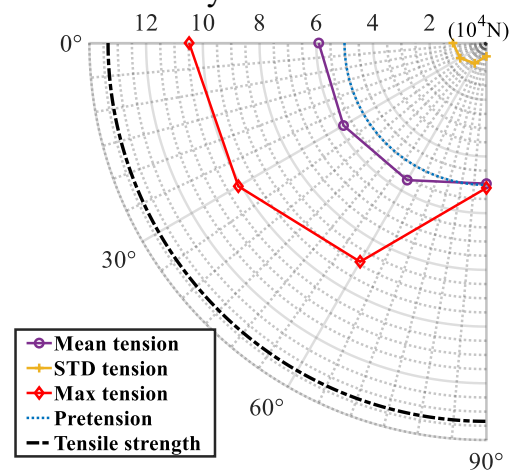

Fig 10. Wire tension for different wind-wave directions 


\section{CONCLUSIONS}

In this study, a numerical model has been established for simulation of a newly developed offshore overhead power transmission system, the TLP-wire system is designed under extreme environmental condition. Main conclusion is given as follows:

- Lumped parameter method can model the TLP-wire system in an acceptable reliability, and the motion properties from simulation agree well with experimental results of TLP-wire interaction.

- For the triangle type TLP, motion of yaw shows a sudden rise at specific wave directions, which can cause more violent change in the tension of tendons and lead to mooring instability.

- In simulation under extreme environmental condition, the wire and tendons run in a safe range, which means the present design system can stand in extreme environment by self-stability.

- Wind-wave misalignment is important in safety evaluation, more experiments under wind-wave condition should be operated in future research.

\section{REFERENCE}

[1] https://www.ren21.net/reports. Renewables 2020 Global Status Report, (2021)131-138

[2] J. M. Jonkman and P. D. Sclavounos. Development of Fully Coupled Aeroelastic and Hydrodynamic Models for Offshore Wind Turbines, 44th AIAA Aerospace Sciences Meeting and Exhibit, 9-12 January 2006.

[3] E. N. Wayman, P. D. Sclavounos, S. Butterfield, J. Jonkman, and W. Musial. Coupled Dynamic Modeling of Floating Wind Turbine Systems, Offshore Technology Conference, Houston, TX, 1-4 May 2006.

[4] J. Jonkman, S. Butterfield, W. Musial, and G. Scott, Definition of a 5-MW Reference Wind Turbine for Offshore System Development, NREL Report No. TP-500-38060, February 2007.

[5] F. G. Nielsen, T. D. Hanson, and B. Skaare. Integrated Dynamic Analysis of Floating Offshore Wind Turbines, Proceedings of the OMAE2006, 25th International Conference on Offshore Mechanics and Arctic Engineering, Hamburg, Germany, 4-9 June 2006.

[6] https://gwec.net/global-wind-report-2017. Global Wind Report2017: Annual Market, (2017)57-58.

[7] http://Windeurope.org/about-wind/statistics/offshore. Offshore Wind in Europe: Key Trends and Statistics 2019, (2020)

[8] LIU Gang, CAO Jingying, LU Ying. Selection Criteria of High-voltage Submarine Cables for Offshore Wind Farms by Life Cycle Cost, High Voltage Engineering, vol.41, (2015)2674-2680.

[9] Kristen R. Schell, João Claro, Seth D. Guikema. Probabilistic cost prediction for submarine power cable projects, Electrical Power and Energy Systems, vol.90, (2017) 1-9

[10] Hongzhong Zhu, Ruo Luo, Changhong Hu, Joshiro Noda. An Experimental Study on Tower-Wire Interaction for Offshore Overhead Power Transmission Concept. The autumn meeting of The
Japan Society of Naval Architects and Ocean Engineers (JASNAOE), 2020.

[11] Bureau Veritas. Hydrostar for expert's user manual, https://www.docenti.unina.it/webdocenti-be/allegati /materiale-didattico/576434. [accessed on 26/April/ 2019] (2016).

[12] C. Lee, J. Newman, WAMIT user manual, version 7.2, WAMIT, Inc., Chestnut Hill, MA

[13] Yingyi Liu, Yoshida Shigeo, Liang Sun, Junliang Gao. Development of a numerical open-source package for marine hydrodynamics: FinGreen3D. 4th International Exchange and Innovation Conference on Engineering \& Sciences (IEICES), October 19-20, 2017, Fukuoka, Japan.

[14] T. I. Fossen. Handbook of marine craft hydrodynamics and motion control, John Wiley \& Sons, 2011.

[15] Y.M. Low. Frequency domain analysis of a tension leg platform with statistical linearization of the tendon restoring forces. Marine Structures 22 (2009) 480-503

[16] Mostafa. A. Rushdi, Shigeo Yoshida, Tarek N. Dief. Simulation of a Tether of a Kite Power System Using a Lumped Mass Model, 4th International Exchange and Innovation Conference on Engineering \& Sciences (IEICES), 18-19th October, 2018, Fukuoka, Japan

[17] Faltinsen O M. Sea loads on ships and offshore structures. Cambridge University Press (1990)

[18] M.L. Facchinetti, E. de Langrea, F. Biolley. Coupling of structure and wake oscillators in vortexinduced vibrations, Journal of Fluids and Structures, vol. 19, (2004) 123-140

[19] Yang Qu, Andrie.V.Metrikine. A single van der pol wake oscillator model for coupled cross-flow and inline vortex-induced vibrations, Ocean Engineering, vol. 196, (2020)

[20] Hongzhong Zhu, Changhong Hu. A Study on Floating Overhead Power Transmission System for Offshore Energy Development: Design, Modeling and Numerical Analysis. (To be presented)

[21] M Irvine. Cable Structures, Dover Publications, 1992. 